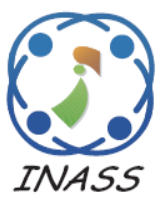

\title{
An Improved Quality Path Selection Approach for Border Gateway Protocol
}

\author{
Shipra Shukla ${ }^{1} \quad$ Mahesh Kumar ${ }^{2 *}$ \\ ${ }^{1}$ Jaypee University of Engineering and Technology, Guna, M.P. India \\ * Corresponding author's Email: mahesh.chahar@gmail.com
}

\begin{abstract}
Route instability causes the frequent changes in path selection on the Internet. A good path selection policy can avoid the repetitive changes of the route by scrutinizing the quality of path in BGP. This work is improving a Stable Route Selection (SRS) approach which has not considered the quality of path in BGP. Nevertheless, the SRS approach selects a route by considering delay and longest path uptime as the foremost criteria, which results in lower throughput and higher end-to-end delay. Consequently, we propose an Improved Stable Path Selection (ISPS) approach, which utilizes the QoS attributes for path selection process competently. For this, we consider available bandwidth, delay and longest path uptime as the QoS attribute for path selection process in BGP. Additionally, in ISPS approach, the BGP routers continuously monitor the queue and traffic flow for selecting the best available path. Subsequently, the proposed ISPS approach adapts the dynamic changes in topology without decreasing the stability of routes and QoS parameters. The simulation results exemplify the usefulness of the proposed ISPS approach over SRS in improving network performance, and yields better results concerning throughput, average end-to-end delay, packet loss rate, and exchanged updates.
\end{abstract}

Keywords: Autonomous systems, BGP, Policy routing, Path selection, QoS routing.

\section{Introduction}

Border Gateway Protocol (BGP) is an interdomain routing protocol. Each autonomous system selects a BGP speaker to share the network layer reachability information with a peer or neighbor BGP speaker. Peer routers establish a BGP session with them to exchange BGP messages. The main objective of BGP is to disseminate network layer reachability information among all peers.

BGP is a path vector routing protocol. Usually, an ISP (Internet Service Provider) connects with more than one inter-domain links to increase the availability of the paths. Every autonomous system computes the degree of preference for all available paths according to path selection policies and selects the best path to propagate such routing decisions to peers.

In a standard BGP route decision process, first and foremost highest local preference of the router is preferred due to business relationships with other ISP. If there is a tie between two paths, then other path selection attributes used to break the tie such as lowest AS path length, lowest origin type, lowest MED, eBGP over iBGP learned paths, lowest IGP cost, lowest router BGP-id and the lowest neighbor IP-address sequentially [1].

Autonomous systems configure BGP routers with diverse policies. The flexible routing policies can increase or decrease the routing stability [2] as well as path availability. The path selection criterion can contribute to networks instability as well. To achieve the traffic engineering goals, IGP metrics such as weight may change repeatedly and misconfiguration of routers can make the availability of path worst [3]. Such type of networks instability cases has been reported in the literature extensively. Therefore, the role of path selection criterion is very vital to improving overall network performance.

In literature, authors have advised various quality path selection policies. BGP routers select the best path considering the stability metrics based on the local stability properties [4], link capacities [5], congestion in the path [6], local guidelines for 
routers [7], and link weight, etc. The authors of [8] used a set of stability metrics based on the local stability properties of BGP routing. These matrices have been applied to decision criterion at the time of route selection. The local QoS routing is used as an alternative of the routing which uses global state information. However, the algorithm uses a predetermined set of candidate paths, which increases the possibility of packet drop during the period of instability.

Bressoud et al. [5] suggested an approach that selects a path according to link capacities for optimum network utilization and load balancing. The algorithm selects a set of border router that maintains the egress bandwidth of the links and monitors traffic flow. The authors claimed that the selected border routers provide optimum utilization of the network. Similarly, the authors of [6] recommended an approach in which every node maintains a set of alternative paths to the destination and each path's current congestion status. The algorithm ranked the path by weight according to current congestion status and selects a path based on weight. However, these approaches increase routing overhead and memory requirement.

Efficient path selection techniques can help achieving high throughput, low end-to-end delay, and low packet loss while, sub-optimal path selection policies can result in route divergence [9] that affects the end to end network performance and quality of service badly [10]. The selection of path based on lowest AS path length reduces end-to-end propagation delay but other network performance metrics such as throughput and percentage of delivered packets does not get regard as criterion for path selection. Hence, it becomes necessary to prefer the high-quality path during path selection.

Therefore, we propose to use the new path selection criteria for path selection using Quality of Service $(\mathrm{QoS})$ metric in an approach called Stable Route Selection (SRS). The main contributions of our approach are as follow.

We propose Improved Stable Path Selection approach (ISPS), a technique for path selection of BGP which does not diminish availability and stability of routes. Moreover, ISPS has the ability to minimize the frequent change of preferred routes, which further reduce packet loss. The proposed approach makes use of QoS metric for the selection of best path among all available paths. In ISPS approach, we have considered available bandwidth, delay and longest path uptime as the QoS metric for path selection process in BGP. Additionally, in the proposed approach, the BGP routers observe and monitor the incoming and outgoing queue and traffic flow for the estimation of available bandwidth and delay. The links are regularly monitored to adapt the dynamic changes in the network topology. For exchanging the QoS metric information, each BGP message includes the QoS metric which subsequently helps in selecting the higher available bandwidth path.

This path selection procedure guarantees low delay. The consideration of QoS metric reduces convergence time, instability and saves the system to suffer from the undesired traffic of updates. ISPS approach significantly improves average throughput and reduces packet loss, average end-to-end delay of the networks in comparison to SRS [2]. In ISPS, we have included QoS metric in path selection procedure described in Section 3.

The remainder sections are as follows. Section 2 covers the problem statement for path selection. Section 3 proposes a solution for the problem; simulation setup has been explained in Section 4, Section 5 evaluates the performance of proposed approach, and Section 7 concludes the paper.

\section{Problem statement}

Godfrey et al. [2] suggested an approach stable route selection (SRS) that defines a path selection policy in BGP. SRS restricts frequent changes in routes that may cause data plane unreliability on the Internet. The approach restrains the load on the control plane of routers as well which exhibits by lots of flexible routing policies that are currently in practice.

In a standard BGP route decision process [1], highest local preference of the router is preferred and then another path selection attributes are considered that is described in Table 1. SRS is an additional step that is placed after deciding local preference of each route in a standard BGP route decision process.

In a network, the Quality of Service attributes improves overall network performance [11]. A good path selection algorithm must consider QoS attributes like path length, delay, and link bandwidth to achieve traffic engineering goals.

SRS approach does not consider the quality of a path that degrades the performance of the entire system during heavy traffic loads. If available bandwidth of a path is very low, then the path may result in failure as well.

In SRS, the main criterion is for path selection is path delay and longest path uptime. Authors have assumed that recently advertised routes are more likely to be withdrawn. To provide the stability in networks the recent advertised routes should not get 
Table1. BGP path selection criterion

\begin{tabular}{|c|l|}
\hline Sr. No. & Action \\
\hline \hline 1 & Highest Local Preference \\
\hline 2 & Lower AS Path Length \\
\hline 3 & Lower Origin \\
\hline 4 & Lower MED \\
\hline 5 & Route learned via eBGP over iBGP \\
\hline 6 & Lower IGP metric to egress point \\
\hline 7 & Lowest Router-id \\
\hline
\end{tabular}

selected. In algorithm, BGP router selected the path with higher path uptime. Additionally, the SRS algorithm did not consider the case when both paths have uptime is not less than delay parameter.

Therefore, in this paper, we propose an improved stable path selection approach (ISPS) which considers path quality. ISPS considers the available bandwidth as a quality of service attribute and provides flexibility in path selection that improves the network's performance in terms of high throughput, low end-to-end delay of the system, minimum exchange of updates in networks and low packet loss. The improved stable path selection approach collects information from intermediate links and considers minimal value in bandwidth along the path [12]. Section 3 describes the proposed solution in detail.

\section{Improved stable path selection (ISPS)}

The proposed approach Improved Stable Path Selection (ISPS), is an improved path selection procedure over SRS. If the BGP routers advertise the change in the path too often then they put an unnecessary burden of exchanged updates.

Hence, to stabilize the networks, network operators should configure the routing policy in such a way which would help to reduce the instability using preferred path policy. The proposed approach does not diminish the availability of the routes. In fact, ISPS selects the quality path among the available paths. ISPS considers the following matrices as a QoS attribute for measuring the path quality.

\subsection{QoS Matrices}

Given a set of autonomous systems, $\mathrm{G}=(\mathrm{V}, \mathrm{E})$ denote the inter-domain topology where $\mathrm{V}$ represents $\mathrm{BGP}$ router, and $\mathrm{E}$ is bidirectional interdomain links. We have assumed that each autonomous system has only one BGP speaker router, which advertises updates. An autonomous system, $\mathrm{AS}_{\mathrm{n}}$, is connected to a set of neighbors $\left\{\mathrm{AS}_{1}\right.$, $\left.\mathrm{AS}_{2} \ldots, \quad \mathrm{AS}_{\mathrm{m}}\right\}$ with bidirectional links. The estimation of delay and available bandwidth metric is done according to the policies of Fault-tolerant robust BGP routing protocol [12].

\subsubsection{Delay metric}

In an abstract topology, there are many available inter-domain paths between two BGP speaker routers. The delay $(\delta)$ of a path $\mathrm{P}$ is the sum of the delays of all the links along the path. $D(l)$ is the sum of processing, propagation and queuing delay of an individual link $l . D(P)$ represents the delay of path $\mathrm{P}$ and $\varphi$ is the set of all links in path $\mathrm{P}$. Then

$$
\delta=D(P)=\sum_{l \in \varphi} D(l)
$$

An upper bound $U_{l}$ is set for each abstract link $l$ in the domain. To meet the traffic engineering goal, it considers the paths whose delay is lower than the upper bound $U_{l}$. The path which does not satisfy this condition will not be considered because they may break traffic regulations of the domain.

\subsubsection{Available link bandwidth metric}

The ISPS monitors a set of links that affect the system. All the links of the topology should be monitored to select the best quality path. There are many available inter-domain paths between two BGP speakers y and $\mathrm{z}$.

$$
S\left((P)_{y, z}\right)=\left\{P \mid P \in S_{(m, n)}^{k}, \quad D(P) \geq U_{l}\right\}
$$

Here, $S_{(m, n)}^{k}$ represents the set of all possible inter-domain paths between two BGP speaker routers. The minimum bandwidth of a physical link along the path is the available bandwidth.

Suppose for link $l$; the bandwidth is $w(l)$, the monitored link of path $\mathrm{P}$ is $l_{B(p)}$ and, the bandwidth of path $\mathrm{P}$ is $w(P)$.

$$
c_{e a}=w(P)=\min _{l \in L_{P}} w(l)=w\left(l_{B(p)}\right)
$$

Here, $L_{P}$ represents the set of physical links along the path and $c_{e a}$ defines the available bandwidth of a path. The QoS metric available link bandwidth propagates with the update. Each BGP speaker has information of $c_{e a}$ that helps to select a path according to path selection procedure (PSP) described in next section. 
The purpose of monitoring a link is that, when the capacity of monitored links reduces, then traffic should move towards other less congested link. Therefore, the link monitoring procedure is used to regulate traffic.

\subsection{Path selection procedure}

Standard BGP [1] applies decision process 1 on Adj-RIB-IN that stores all available paths from neighbors. SRS [2] inserts an additional step in path selection procedure after assigning the route local preference in the standard BGP decision process1 [1]. Proposed ISPS modifies the SRS [2] path selection procedure. The approach applies path selection procedure on two available good paths $\mathrm{P}_{1}$ and $\mathrm{P}_{2}$ reachable to a destination $\mathrm{p}$. Both the paths $\mathrm{P}_{1}$ and $\mathrm{P}_{2}$ are constantly available during the period of instability.

The path selection procedure $\operatorname{PSP}\left(\mathrm{P}_{1}, \mathrm{P}_{2}\right)$ is applied on Adj-RIB-IN [1]. The ISPS selects a quality path based on available bandwidth when none of the paths is selected in step $1 \& 2$.

PSP $\left(\mathrm{P}_{1}, \mathrm{P}_{2}\right)$ selects the best path between path $\mathrm{P}_{1}$ and $\mathrm{P}_{2}$ when uptime cannot help selecting the best path. The paths $\mathrm{P}_{1}, \mathrm{P}_{2}$ have uptimes $\geq \delta$ (delay) and available bandwidths are $c_{e a_{1}}, c_{e a_{2}}$ respectively. In step one and two, if $\mathrm{P}_{1}$ and $\mathrm{P}_{2}$ have uptime greater than or equal to $\delta$, then PSP selects the preferred path. Routers advertise the new path whenever the best path changes in the router's routing table.

If the selected paths change very frequently then they cause a high amount of message overhead and create instability in the networks. Hence, the main idea is to avoid the selection of newly advertised path to maintain the stability of the networks.

If both the paths have uptime greater than or equal to $\delta$ then step three introduces new criterion of comparing paths based on available bandwidths $c_{e a_{1}}$, $c_{e a_{2}}$ and selects the path having a higher available bandwidth $\left(c_{e a}\right)$.

If both path $\mathrm{P} 1$ and $\mathrm{P} 2$ have the equal available bandwidth, and any of the paths has not been selected in step three then in step four PSP stays with the previously selected path. Else if none of the paths has been selected till step four, and then step five selects the path which has shorter AS path. Moreover, if none of the paths has been selected up to step five, then PSP selects the path with higher uptime [2].

The path selected by PSP will be installed in LOC-RIB [1] by decision process2. Adj-RIB-OUT [1] accumulates all advertisable paths and disseminates paths to its neighbor.

\section{Algorithm 1: Path Selection Procedure}

\section{Begin Procedure PSP $\left(\mathbf{P}_{1}, \mathbf{P}_{2}\right)$}

1) If $\mathrm{P}_{1}$ has been up for the time $\geq \delta$ and Preference of $\mathrm{P}_{1}>$ Preference of $\mathrm{P}_{2}$ End If Then select $P_{1}$

2) If $\mathrm{P}_{2}$ has been up for the time $\geq \delta$ and Preference of $\mathrm{P}_{2}>$ Preference of $\mathrm{P}_{1}$

End if

Then select $\mathrm{P}_{2}$

3) Else if, select the route on the basis of path quality

3.1) If $c_{e a_{1}}>c_{e a_{2}}$ and Preference of $\mathrm{P}_{1}>$ Preference of $\mathrm{P}_{2}$

Then select $P_{1}$

End if

3.2) Else If $c_{e a_{2}}>c_{e a_{1}}$ and Preference of $\mathrm{P}_{2}$ $>$ Preference of $\mathrm{P}_{1}$

Then select $\mathrm{P}_{2}$

\section{End if}

End if

4) Else if, one of the paths has been selected between $\mathrm{P}_{1}$ and $\mathrm{P}_{2}$ End if

Then stay with that route

5) Else If, select the route between $P_{1}$ and $P_{2}$ on the basis of AS path length

Then choose the route with shorter AS path length.

End if

6) Else If, select the route between $P_{1}$ and $P_{2}$ on the basis of uptime of path

Then choose the path with higher uptime. End If

\section{End Procedure}

\section{Simulation Setup}

This section describes the simulation setup. All the experiments are simulated in NS2 version 2.34 [13]. NS-BGP 2.0 [14] is an extension of NS2 simulator that is used for BGP simulation. The proposed approach Improved Stable Path Selection (ISPS) has been compared with Stable Route Selection (SRS) [2]. 


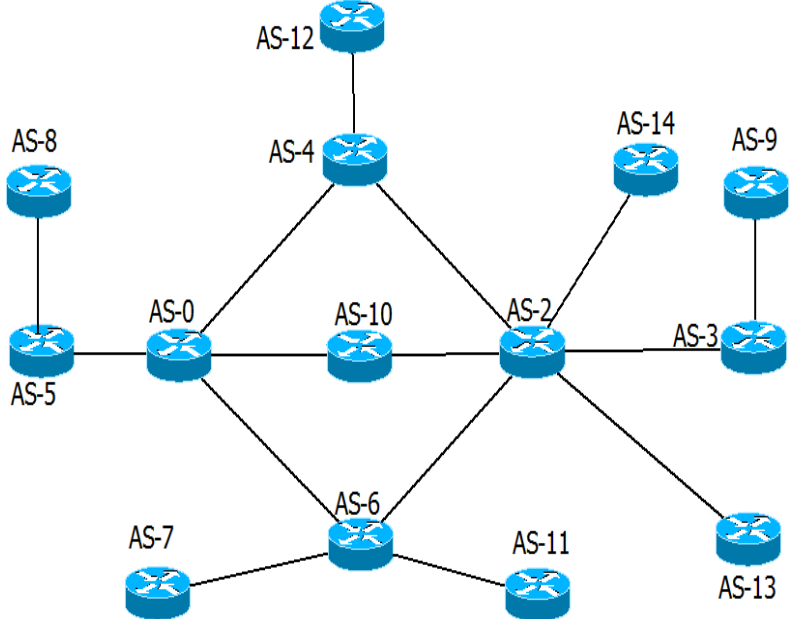

Figure.1 Simulation topology

The simulated topology of 14 nodes has been shown in Fig. 1. The topology is similar to topology presented in CAIDA project [15]. We have assumed each autonomous system (AS) as one BGP speaker router that represents a node. The IP addresses for each AS range from 10.0.0.1 to 10.0.14.1.

All links have been up at the same time 0.1 milliseconds (ms). Table 2 represents the assigned link bandwidth and delay of each link. Source node AS5 generates CBR traffic to destination node AS3.

Table 2. Link bandwidth (Mbps) and link delay of all links

\begin{tabular}{|l|l|l|l|}
\hline $\begin{array}{l}\text { Sr. } \\
\text { No. }\end{array}$ & Link & $\begin{array}{l}\text { Link } \\
\text { bandwidth } \\
\text { (Mbps) }\end{array}$ & $\begin{array}{l}\text { Link delay } \\
(\mathbf{m s})\end{array}$ \\
\hline 1 & $\mathrm{AS}_{0}-\mathrm{AS}_{4}$ & $1 \mathrm{Mbps}$ & $20 \mathrm{~ms}$ \\
\hline 2 & $\mathrm{AS}_{0}-\mathrm{AS}_{10}$ & $5 \mathrm{Mbps}$ & $100 \mathrm{~ms}$ \\
\hline 3 & $\mathrm{AS}_{0}-\mathrm{AS}_{6}$ & $10 \mathrm{Mbps}$ & $1 \mathrm{~ms}$ \\
\hline 4 & $\mathrm{AS}_{0}-\mathrm{AS}_{5}$ & $10 \mathrm{Mbps}$ & $1 \mathrm{~ms}$ \\
\hline 5 & $\mathrm{AS}_{5}-\mathrm{AS}_{8}$ & $1 \mathrm{Mbps}$ & $10 \mathrm{~ms}$ \\
\hline 6 & $\mathrm{AS}_{6}-\mathrm{AS}_{7}$ & $1 \mathrm{Mbps}$ & $10 \mathrm{~ms}$ \\
\hline 7 & $\mathrm{AS}_{6}-\mathrm{AS}_{11}$ & $1 \mathrm{Mbps}$ & $10 \mathrm{~ms}$ \\
\hline 8 & $\mathrm{AS}_{4}-\mathrm{AS}_{2}$ & $1 \mathrm{Mbps}$ & $100 \mathrm{~ms}$ \\
\hline 9 & $\mathrm{AS}_{10}-\mathrm{AS}_{2}$ & $5 \mathrm{Mbps}$ & $1 \mathrm{~ms}$ \\
\hline 10 & $\mathrm{AS}_{6}-\mathrm{AS}_{2}$ & $10 \mathrm{Mbps}$ & $1 \mathrm{~ms}$ \\
\hline 11 & $\mathrm{AS}_{2}-\mathrm{AS}_{14}$ & $1 \mathrm{Mbps}$ & $10 \mathrm{~ms}$ \\
\hline 12 & $\mathrm{AS}_{2}-\mathrm{AS}_{3}$ & $10 \mathrm{Mbps}$ & $1 \mathrm{~ms}$ \\
\hline 13 & $\mathrm{AS}_{2}-\mathrm{AS}_{13}$ & $1 \mathrm{Mbps}$ & $10 \mathrm{~ms}$ \\
\hline 14 & $\mathrm{AS}_{3}-\mathrm{AS}_{9}$ & $1 \mathrm{Mbps}$ & $10 \mathrm{~ms}$ \\
\hline
\end{tabular}

A flow of data packets varies from source to destination router. The constant delay is inserted at each link. Traffic rate varies between 1.0 Mbps and $1.6 \mathrm{Mbps}$.

We assumed that all links are up during path selection and there is no failure of links during path selection. The source AS sends the traffic to the destination AS.

The estimation of available bandwidth is done at each router. BGP routers of intermediate AS share the available bandwidth information with peers using the BGP updates. The paths remain consistent at any point of time.

BGP routers filter the sets of path on each step using attributes to select a best path. By applying the filter on each step it gets a set of the path. We altered the BGP decision process policy on routers according to the proposed approach presented in algorithm 1. The constraints defined in section 3 are applied between step 1 and 2 in table 1 .

\section{Results and discussions}

This section presents the obtained network performance results under different circumstances. To evaluate our approach, network performance matrices throughput, average end-to-end delay, percentage of delivered packets, and exchanged updates between ASes have been recorded. The existing BGP policy SRS [2] is compared with the proposed approach ISPS.

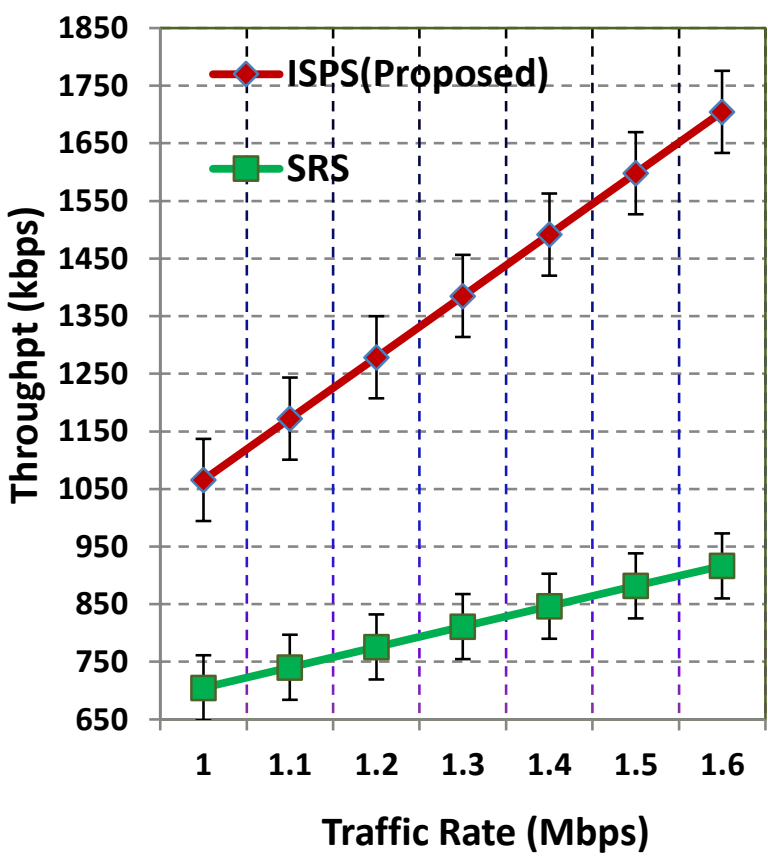

Figure. 2 Rate vs. throughput 


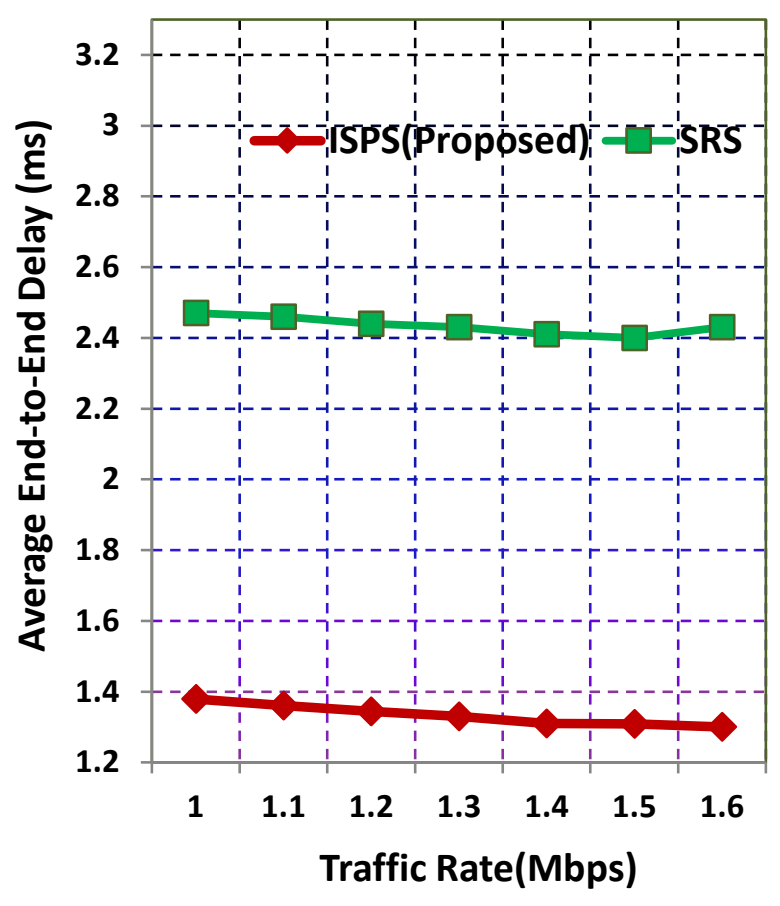

Figure. 3 Rate vs. average end-to-end delay

Fig. 2 shows that the throughput increases with the increase of traffic rate. In ISPS, throughput picks up from $1065.65 \mathrm{kbps}$ to $1704.51 \mathrm{kbps}$, while in SRS, throughput raises from $704.92 \mathrm{kbps}$ to 916.43 kbps.

Hence, ISPS improves throughput significantly in between $33.8 \%$ to $46.2 \%$ (approx.) as compared to SRS. To assure the $95 \%$ confidence level, we obtained the confidence interval of throughput which lies between 754.56to 867.46 kbps in SRS while 1313.801 to1456.361 kbps in ISPS.

Fig. 3 compares the average end-to-end delay of both approaches in networks. We have estimated average end-to-end delay from source to destination at traffic rates (1 to $1.6 \mathrm{Mbps}$ ). Our approach ISPS produced lower delay as compared to SRS. Fig. 4 represents the trend of delay in packet delivery to destination when traffic moves at the rate of 1.6 Mbps in both the approaches. We have shown an interval where proposed approach exhibits much lower delay than SRS.

Our approach selects the path based on high available bandwidth and low delay of a path. Hence, it reduces the probability of packet loss. Fig 5 compares the percentage of delivered packets in both approaches. In comparison to increment in number of the packet sent, the packet loss is quite high in SRS. The number of packets sent is 327751, and the packet loss is 37530 when traffic rate moves at the rate of $1.6 \mathrm{Mbps}$.

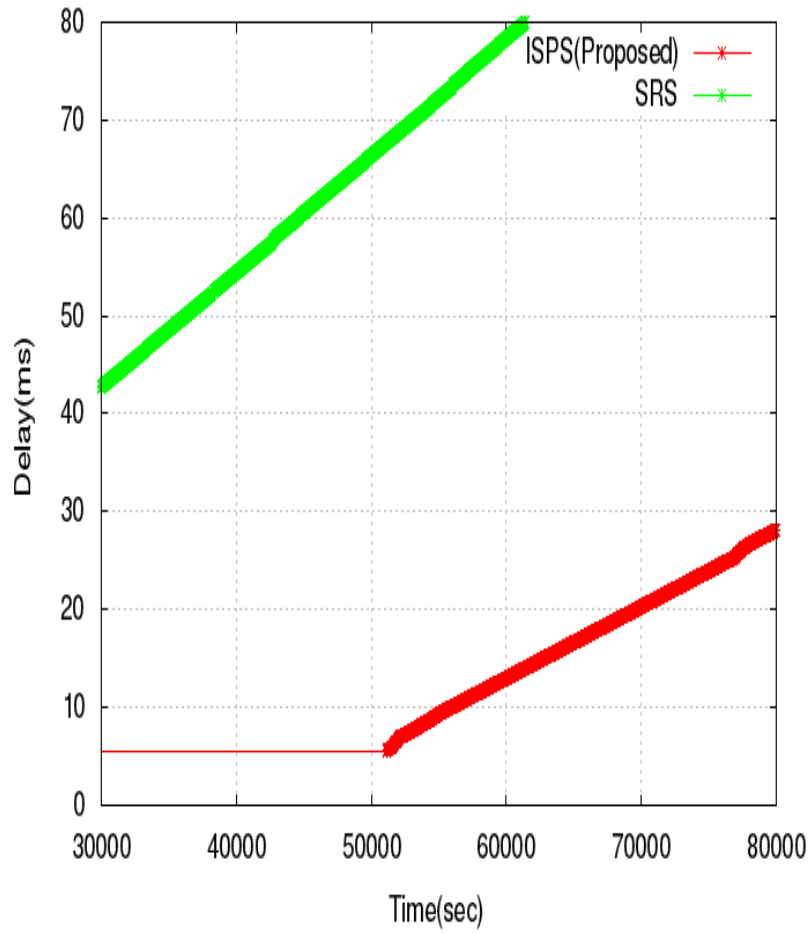

Figure. 4 Comparison of delay of packets (ms) in SRS and ISPS

The loss percentage varied between 0 to $12 \%$ (approx.) in SRS but our approach (ISPS) produced almost no loss of packets due to path selection policy.

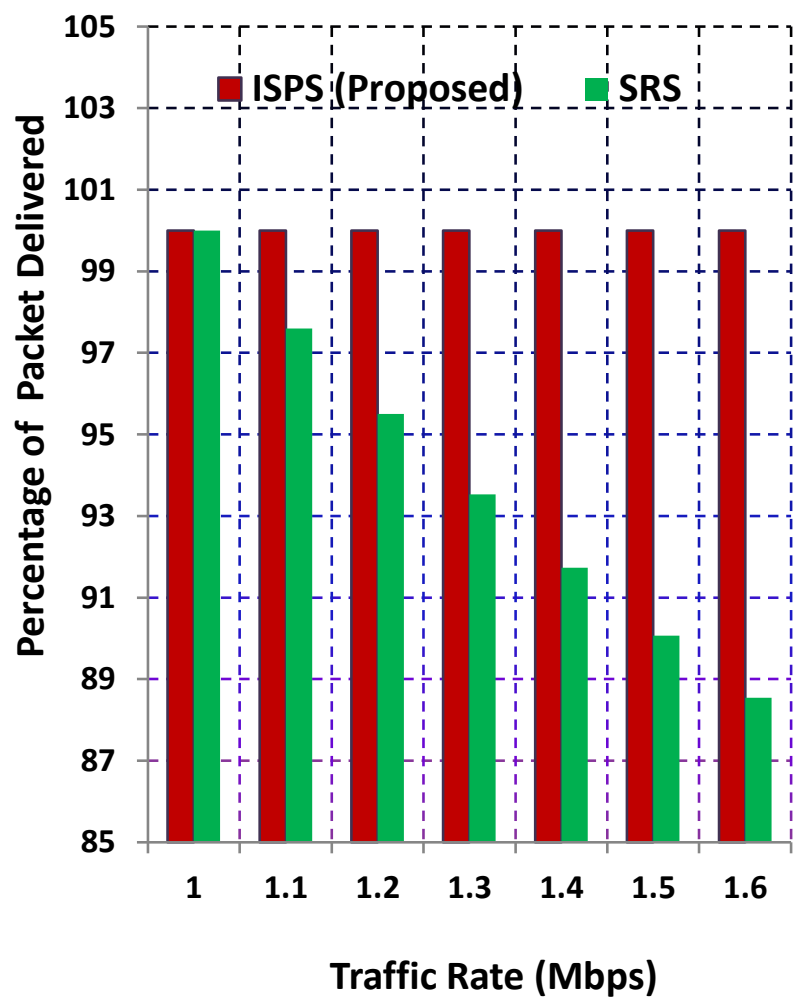

Figure. 5 Rate vs. percentage of delivered packets 
BGP routing table of $\mathrm{AS}_{0}$

Local router ID is 10.0.0.1

Status codes: * valid, > best, i - internal. Network Next Hop Path

*> 10.0.0.0/24 self

*> 10.0.1.0/24 10.0.10.1/32 AS10

*> 10.0.2.0/24 10.0.4.1/32 AS 4AS 2

$*>10.0 .3 .0 / 24 \quad 10.0 .4 .1 / 32 \quad$ AS 4AS 2 AS 3

*> 10.0.4.0/24 10.0.4.1/32 AS 4

*> 10.0.5.0/24 10.0.5.1/32 AS 5

*> 10.0.6.0/24 10.0.6.1/32 AS 6

* $>30.30 .3 .0 / 24 \quad 10.0 .4 .1 / 32 \quad$ AS 4AS 2AS 3

*> 50.50.3.0/24 10.0.4.1/32 AS 4AS 2AS 3

Fig.6. Routing table of $\mathrm{AS}_{0}$ in SRS approach

The results presented in Figs. 2 to 5 show that the throughput, packet delivery ratio is higher and average end-to-end delay is lower in ISPS compared to SRS. The path selection criterion of both approaches is different. The idea of forwarding traffic towards high available bandwidth path is the major reason behind the improved performance of the proposed ISPS approach regarding higher throughput and lower average end-to-end delay (as shown in Figs. 2, 3, and 4). However, SRS approach does not take care of available bandwidth of a path, and forwards the traffic towards a path having higher path uptime. Moreover, in case of tie between path uptime (i.e., paths having same path uptime), SRS selects a path which have lowest router id.

To confirm that our approach followed the modified path selection criterion, we have shown the routing tables of $\mathrm{AS}_{0}$. There are three paths to reach the traffic from $\mathrm{AS}_{0}$ to $\mathrm{AS}_{3}$. The routing table of $\mathrm{AS}_{0}$ can tell us which path got selected in both the approaches. Therefore, we have shown the routing table of $\mathrm{AS}_{0}$ in SRS and ISPS respectively in Figs. 6 and 7.

\begin{tabular}{|c|c|c|}
\hline \multicolumn{3}{|c|}{ BGP routing table of $\mathrm{AS}_{0}$} \\
\hline \multicolumn{3}{|c|}{ Status codes: ${ }^{*}$ valid, > best, i - internal. } \\
\hline Network & Next Hop & Path \\
\hline$*>10.0 .0 .0 / 24$ & self & \\
\hline$*>10.0 .1 .0 / 24$ & $10.0 .10 .1 / 32$ & AS 10 \\
\hline$*>10.0 .2 .0 / 24$ & $10.0 .6 .1 / 32$ & AS 6AS 2 \\
\hline$*>10.0 .3 .0 / 24$ & $10.0 .6 .1 / 32$ & AS 6 AS 2 AS 3 \\
\hline$*>10.0 .4 .0 / 24$ & $10.0 .4 .1 / 32$ & AS 4 \\
\hline$*>10.0 .5 .0 / 24$ & $10.0 .5 .1 / 32$ & AS 5 \\
\hline$*>10.0 .6 .0 / 24$ & $10.0 .6 .1 / 32$ & AS 10 AS 2 AS 6 \\
\hline$*>30.30 .3 .0 / 24$ & $10.0 .6 .1 / 32$ & AS 6 AS 2 AS 3 \\
\hline$*>50.50 .3 .0 / 24$ & $10.0 .6 .1 / 32$ & AS 6AS 2AS 3 \\
\hline
\end{tabular}

Figure. 7 Routing table of $\mathrm{AS}_{0}$ in ISPS approach

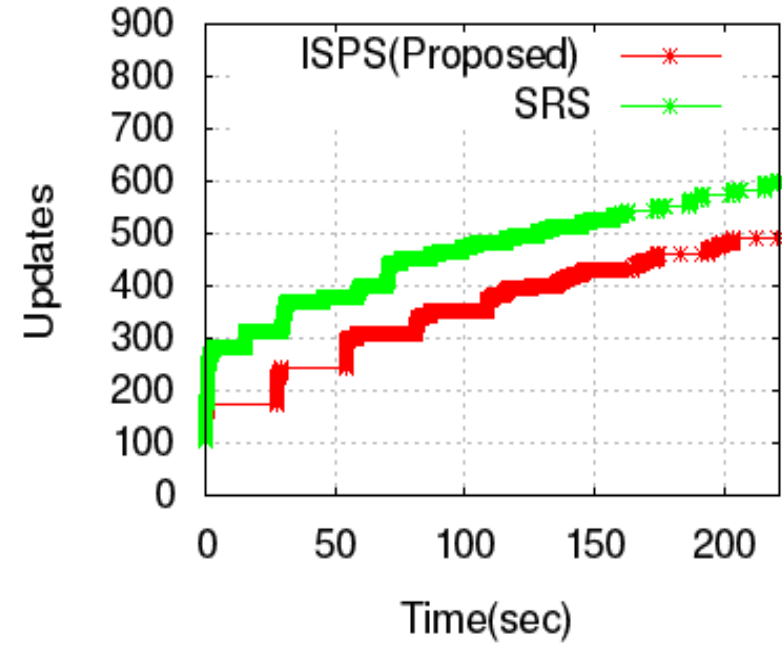

Figure. 8 Comparison of exchanged updates of ISPS and SRS

The highlighted blue color rows depict the path selected for the networks 10.0.3.0/24 and 30.30.3.0/24 which are advertised by $\mathrm{AS}_{3}$. SRS selects the shortest path $\mathrm{AS}_{4} \mathrm{AS}_{2} \mathrm{AS}_{3}$ by longest path uptime while ISPS selects the path $\mathrm{AS}_{6} \mathrm{AS}_{2} \mathrm{AS}_{3}$ based on available bandwidth. Initially, the available bandwidth of the path $\mathrm{AS}_{4} \mathrm{AS}_{2} \mathrm{AS}_{3}$ is approximately $1 \mathrm{Mbps}$ which is lower than the available bandwidth of the path $\mathrm{AS}_{6} \mathrm{AS}_{2} \mathrm{AS}_{3}$ which is around $10 \mathrm{Mbps}$. Hence, the selection of the path $\mathrm{AS}_{4} \mathrm{AS}_{2} \mathrm{AS}_{3}$ leads to high packet loss in networks as shown in Fig. 5.

Our approach does not diminish the stability of the networks. To confirm the stability improvement of ISPS, we recorded the network stability metric which is the number of exchanged updates within a specific time interval. Fig. 8 shows the exchanged updates between 0 to 250 seconds in both the approaches. The number of exchanged updates in ISPS are approximately $10 \%$ lesser than SRS. Hence, ISPS maintains stability as well.

\section{Conclusions and future work}

In this paper, we propose, ISPS, which modifies the path selection process using QoS metrics efficiently in BGP. The approach selects the best quality of path considering available bandwidth, delay, and path uptime as the QoS metric. Additionally, in the approach, BGP routers continuously monitor the queue and traffic flow in network. Specifically, compared with SRS, ISPS offers $41 \%$ higher throughput relatively with $45 \%$ lesser average end-to-end delay, and minimizes the packet loss rate up to $12 \%$ approximately. Further, in ISPS, $10 \%$ lesser update messages are exchanged between autonomous systems as compared to SRS. 
This paper points to an interesting direction of future work. The performance evaluation has been done by considering single interface equipped end devices between two autonomous systems. Nevertheless, the novel idea of forwarding through multi-interface equipped end devices (multihoming) can seriously affect the performance of network. Additionally, the abrupt intra-domain path selection policies of BGP can deflect the selected routes as well.

\section{References}

[1] Y. Rekhter, T. Li, and S. Hares, A Border Gateway Protocol, RFC 1771, 1995

[2] P. B. Godfrey, I. Haken, Y. Singer, and S. Shenker, "Stabilizing Route Selection in BGP", IEEE/ACM Transactions on Networking, Vol. 23, No. 1, pp. $282-299,2015$.

[3] A. R. Rivera, K.W. Chin, and S. Soh, "A Novel Framework To Mitigate The Negative Impacts Of Green Techniques On BGP", Journal of Network and Computer Applications, Vol. 48, No.3, pp.22-34, 2015.

[4] X. Yuan and A. Saifee, "Path Selection Methods for Localized Quality of Service Routing", In: Proc. of 10th International Conference on Computer Communications and Networks, Scottsdale, AZ, USA, pp. 102-110, 2001.

[5] T. C. Bressoud, R. Rastogi, and M. A. Smith, "Optimal Configuration for BGP Route Selection", In: Proc. of 22nd Annual Joint Conference of the IEEE Computer and Communications, San Francisco, CA, USA, pp. 916-926, 2003.

[6] L. Yang and G. N. Rouskas, "Adaptive Path Selection in OBS Networks", Journal of Lightwave Technology, Vol. 24, No. 8, pp. 3002-3011, 2006.

[7] W. Feng, F. Chang, W. Feng, and J. Walpole, "Provisioning on-line games: A traffic analysis of a busy Counter-Strike server", In: Proc. of Internet Measurement Workshop, NY, USA, pp. 151-156, 2002.

[8] D. Papadimitriou, A. C. Aparicio, and F. Coras, "Stability metrics and criteria for path-vector routing", In: Proc. of International Conference on Computing, Networking and Communications, San Diego, CA, USA, pp. 1036- 1042, 2013.

[9] L. Xiao, J. Wang, K.S. Lui, and K. Nahrstedt, "Advertising Interdomain QoS Routing Information", IEEE Journal On Selected Areas In Communications, Vol. 22, No. 10, pp. 19491964, 2004.
[10] C. Labovitz, A. Ahuja, A. Bose, and F. Jahanian, "Delayed Internet routing convergence", Computer Communication Review, Vol.30, No. 4, pp. 175-187, 2000.

[11] D. Perouli, T. G. Griffin, O. Maennel, and S. Fahmy, "Detecting Unsafe BGP Policies in a Flexible World", In: Proc. of 20th IEEE International Conference on Network Protocols (ICNP), Austin, TX, USA, pp. 1 10, 2012.

[12] M. Kumar and S. Kumar, "An approach to stabilize interdomain routing protocol after failure", Computers and Electrical Engineering, Vol. 39, No. 2, pp. 237-245, 2013.

[13] T. Issariyakul and E. Hossain, Introduction to network simulator NS2, Springer Science \& Business Media, pp. 1-18, 2012.

[14] M. R. Sahraei, Integration of $n s-B G P$ with $n s-$ 2.34., Online Report, Dec 2009.

[15] CAIDA,2017,[Online].Available:www.caida.or g/tools/measurement/mantra/\#viz, Accessed on June 2017. 